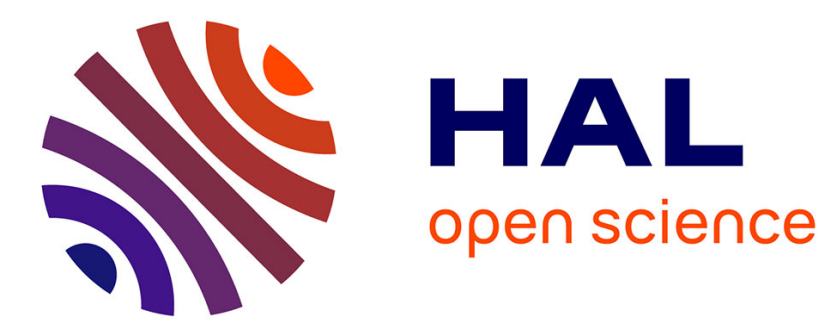

\title{
A quasi-static approach to the stability of the path of heavy bodies falling within a viscous fluid
}

\author{
David Fabre, Pauline Assemat, Jacques Magnaudet
}

\section{To cite this version:}

David Fabre, Pauline Assemat, Jacques Magnaudet. A quasi-static approach to the stability of the path of heavy bodies falling within a viscous fluid. Journal of Fluids and Structures, 2011, vol. 27, pp. 758-767. 10.1016/j.jfluidstructs.2011.03.013 . hal-00908806

\section{HAL Id: hal-00908806 https://hal.science/hal-00908806}

Submitted on 25 Nov 2013

HAL is a multi-disciplinary open access archive for the deposit and dissemination of scientific research documents, whether they are published or not. The documents may come from teaching and research institutions in France or abroad, or from public or private research centers.
L'archive ouverte pluridisciplinaire HAL, est destinée au dépôt et à la diffusion de documents scientifiques de niveau recherche, publiés ou non, émanant des établissements d'enseignement et de recherche français ou étrangers, des laboratoires publics ou privés. 


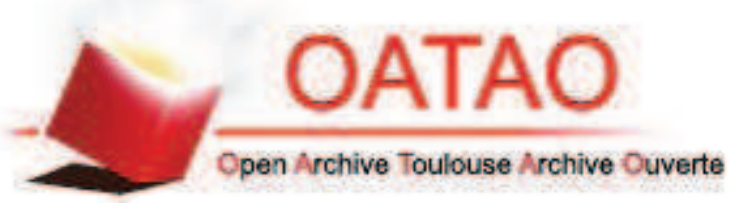

Open Archive TOULOUSE Archive Ouverte (OATAO)

OATAO is an open access repository that collects the work of Toulouse researchers and makes it freely available over the web where possible.

This is an author-deposited version published in : http://oatao.univ-toulouse.fr/ Eprints ID : 10261

To link to this article : DOI:10.1016/j.jfluidsstructs.2011.03.013

URL : http://dx.doi.org/10.1016/j.jfluidstructs.2011.03.013

To cite this version : Fabre, David and Assemat, Pauline and Magnaudet, Jacques A quasi-static approach to the stability of the path of heavy bodies falling within a viscous fluid. (2011) Journal of Fluids and Structures, vol. 27 $\left(n^{\circ}\right.$ 5-6). pp. 758-767. ISSN 0889-9746

Any correspondance concerning this service should be sent to the repository administrator: staff-oatao@listes-diff.inp-toulouse.fr 


\title{
A quasi-static approach to the stability of the path of heavy bodies falling within a viscous fluid
}

\author{
D. Fabre*, P. Assemat, J. Magnaudet
}

Institut de Mécanique des Fluides, Université de Toulouse and CNRS, Allée Camille Soula, F-31400 Toulouse, France

Keywords:

Fluid-solid interaction

Linear stability

Fluttering mode

Path instability

Numerical simulation

\begin{abstract}
A B S T R A C T
We consider the gravity-driven motion of a heavy two-dimensional rigid body freely falling in a viscous fluid. We introduce a quasi-static linear model of the forces and torques induced by the possible changes in the body velocity, or by the occurrence of a nonzero incidence angle or a spanwise rotation of the body. The coefficients involved in this model are accurately computed over a full range of Reynolds number by numerically resolving the Navier-Stokes equations, considering three elementary situations where the motion of the body is prescribed. The falling body is found to exhibit three distinct eigenmodes which are always damped in the case of a thin plate with uniform mass loading or a circular cylinder, but may be amplified for other geometries, such as in the case of a square cylinder.
\end{abstract}

\section{Introduction}

The fall of tree leaves, seeds or paper cards in air has ever been a subject of great fascination. Depending on their geometry and mass but also on the initial conditions of their release, such bodies can follow a straight vertical path, flutter about the vertical or tumble along an inclined path, a motion also known as autorotation (Lugt, 1983). The rational study of these complex motions in the framework of fluid dynamics principles probably goes back to Maxwell (1854) and involved other great names such as Helmholtz, Kelvin and Kirchhoff (see Lamb, 1945). Since then, the description, understanding and prediction of these various types of path and of the transitions between them has remained a subject of constant interest. Most of the modern experimental studies on this subject have been carried out with three-dimensional bodies such as disks of various thicknesses (Willmarth et al., 1964; Field et al., 1997; Fernandes et al., 2007) and spheres (Horowitz and Williamson, 2010). Nevertheless some experiments specifically focused on nearly two-dimensional bodies such as plastic or metal strips (Belmonte et al., 1998; Mahadevan et al., 1999; Andersen et al., 2005b). As they significantly reduce the computational cost, such two-dimensional geometries have also been recently the subject of several numerical investigations, mostly at high Reynolds number (Jones and Shelley, 2005; Andersen et al., 2005a; Jin and Xu, 2008; Kolomenskiy and Schneider, 2010). In parallel, a series of analytical two-dimensional models based on the force/torque balance on the body emerged (Tanabe and Kaneko, 1994; Mahadevan, 1996; Belmonte et al., 1998; Pesavento and Wang, 2004; Andersen et al., 2005a). These models were mostly designed to predict path transitions in highly nonlinear regimes, especially that between fluttering and tumbling. This is why they mostly differ in the way they take into account the nonlinearities involved in the aerodynamic drag, lift and torque. The very first transition between the vertical path and the fluttering regime has received less attention and corresponding models only start to emerge (Ern et al., 2009). Although

\footnotetext{
* Corresponding author at: Institut de Mécanique des Fluides de Toulouse, Université de Toulouse, Allée Camille Soula, F-31400 Toulouse, France.
} E-mail address: david.fabre@imft.fr (D. Fabre). 
the typical flow Reynolds number in the latter case is significantly lower than those associated with subsequent transitions, the thick boundary layer and wake that interact with the body make the modelling of the fluid force and torque pretty difficult. In particular, high-Reynolds-number concepts and results from potential flow theory hardly apply.

The present investigation is a step forward in the understanding of this first transition and aims at providing a rational approach to the coupled fluid-body stability problem. For this purpose we derive in Section 2 a general linear model of forces and torques induced by small-amplitude body motions to which we apply classical methods of linear stability theory. In Section 3 we show that the six coefficients entering the model can be accurately determined by numerically resolving the Navier-Stokes equations to obtain the flow field around the body with suitable prescribed conditions at infinity. We present the results of the complete approach for various body shapes in Section 4 . The results of the model are compared in Section 5 with those obtained with a more general global stability approach developed in a companion paper. Section 6 summarizes the results and discuss their implications regarding the nature of the transition to fluttering.

\section{Quasi-static model : description and discussion}

\subsection{Notations and definitions}

In what follows we consider a 2D body with a mass $m$ (actually a mass per unit span), a moment of inertia $I=c_{I} m d^{2}$ and a volume (actually a cross sectional area) $\mathcal{V}$. The coefficient $c_{I}$ depends upon the geometry and mass repartition of the body. ${ }^{1}$

The body is bounded by a contour $\mathcal{S}$ and the unit normal $\mathbf{n}$ points into the fluid. As shown in Fig. 1(a), we introduce three reference frames. The first of these, $\left(O, \mathbf{x}_{\mathbf{0}}, \mathbf{y}_{\mathbf{0}}, \mathbf{z}\right)$, is associated with the laboratory reference frame (assumed inertial) with $-\mathbf{x}_{\mathbf{0}}$ denoting the direction of gravity $\mathbf{g}$. The reference frame $\left(G, \mathbf{x}_{\mathbf{b}}, \mathbf{y}_{\mathbf{b}}, \mathbf{z}\right)$ is attached to the body, assuming that the direction $\mathbf{x}_{\mathbf{b}}$ defines a symmetry plane. Finally $\left(G, \mathbf{x}_{\mathbf{a}}, \mathbf{y}_{\mathbf{a}}, \mathbf{z}\right)$ denotes the aerodynamic (or Frenet) reference frame defined so that $\mathbf{x}_{\mathbf{a}}$ is aligned with the instantaneous velocity of the body $\mathbf{U}$, i.e. $\mathbf{U}=U \mathbf{x}_{\mathbf{a}}$.

In what follows $\theta$ denotes the inclination of the body with respect to the vertical (i.e. the angle between $\mathbf{x}_{\mathbf{0}}$ and $\mathbf{x}_{\mathbf{b}}$ ), $\alpha$ the angle of incidence between the axis of the body and its trajectory (i.e. that between $\mathbf{x}_{\mathbf{a}}$ and $\mathbf{x}_{\mathbf{b}}$ ), and $\gamma$ is the slope of the trajectory with respect to the vertical. These angles obey the relation $\theta=\alpha+\gamma$. Finally, the rotation of the body with respect to the inertial reference frame is $\boldsymbol{\Omega}=\omega \mathbf{z}$, with $\omega=\dot{\theta}$.

The conventions used here strongly resemble those employed in flight mechanics (e.g. McCormick, 1995). However, there is a few significant differences. First, the slope $\gamma$ is defined with respect to the vertical direction while in the aeronautical context it generally refers to the horizontal direction which is assumed to be the normal flight condition. Second, when considering a slender body, $\alpha$ becomes the angle between the minor axis and the velocity, so that a zero incidence means that the body major axis is perpendicular to the flow while for a wing, zero incidence generally means that the chord is aligned with the flow.

The body motion is governed by Newton's equations:

$$
\begin{aligned}
& m \frac{d \mathbf{U}}{d t}=\left(m-\rho_{f} \mathcal{V}\right) \mathbf{g}+\mathbf{F}, \\
& I \dot{\omega}=M .
\end{aligned}
$$

The terms on the right-hand side are the buoyancy-corrected gravity and the aerodynamic force and torque whose mathematical expression as function of the flow parameters will be discussed later. When expressed in the aerodynamic axes, the acceleration and the aerodynamic force take the form

$$
\frac{d \mathbf{U}}{d t}=\dot{U} \mathbf{x}_{\mathbf{a}}+U \dot{\gamma} \mathbf{y}_{\mathbf{a}} ; \quad \mathbf{F}=-D \mathbf{x}_{\mathbf{a}}-L \mathbf{y}_{\mathbf{a}},
$$

where $D$ and $L$ are by definition the drag and lift.

Following the classical convention in aerodynamics, we introduce the drag, lift and torque coefficient $C_{d}, C_{l}$ and $C_{m}$ so that

$$
D=1 / 2 \rho_{f} C_{d} d U^{2} ; \quad L=1 / 2 \rho_{f} C_{l} d U^{2} ; \quad M=1 / 4 \rho_{f} C_{m} d^{2} U^{2},
$$

where $d$ is the body diameter and $\rho_{f}$ the density of the fluid.

Note that we did not split the fluid force and torque into added-mass (i.e. irrotational) and vortical contributions, so that both series of effects are in principle contained in the coefficients we just introduced. Nevertheless, since we focus on the "heavy" limit where accelerations are assumed small added-mass effects are expected to be negligibly small in the present context. The situation would be dramatically different if we were considering "light" bodies, especially rising gas bubbles. In such a case, the generalized Kirchhoff equations where added-mass contributions are made explicit would provide a much better starting point (Ern et al., 2009).

${ }^{1}$ Plate with uniform loading $c_{I}=1 / 12$; with elliptic loading $c_{I}=1 / 16$; loaded at both extremities $c_{I}=1 / 4$. Square cylinder: $c_{I}=1 / 6$; circular cylinder: $c_{I}=1 / 8$. 

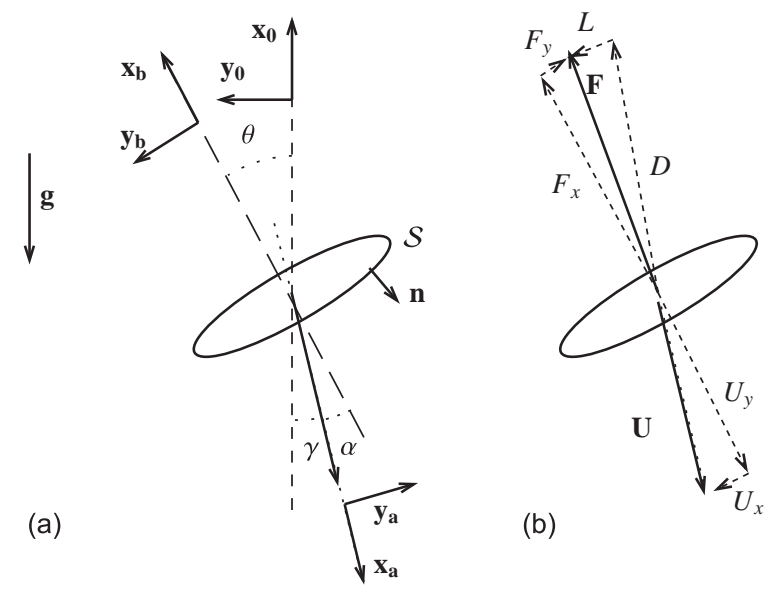

(b)

Fig. 1. Schematic view of the problem and notations. (a) the three system of axes; (b) projection of the force and velocity onto the aerodynamical and body's system of axes.
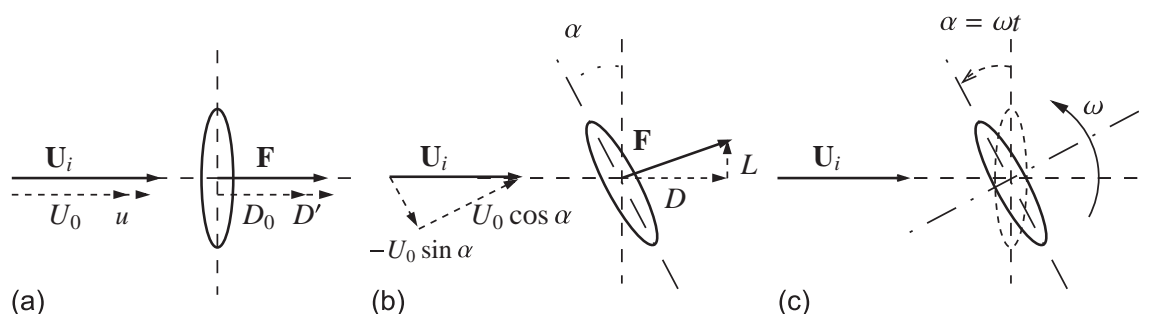

(c)

Fig. 2. The three elementary situations considered for computing the six coefficients of the model. (a) $D_{0}$ and $D_{, u}$; (b) $C_{l, \alpha}$ and $M_{, \alpha}$; (c) $C_{l, \omega}$ and $M_{, \omega}$.

\subsection{The base solution: steady vertical fall}

As the body is assumed to exhibit a geometrical symmetry, the equations of motion obviously admit a solution corresponding to a steady vertical fall with zero incidence. This situation corresponds to $\mathbf{U}=U_{0} \mathbf{x}_{\mathbf{a}}=-U_{0} \mathbf{x}_{\mathbf{b}}=-U_{0} \mathbf{x}_{\mathbf{0}}$. In this case the force balance reduces to

$$
D_{0}=(m-\rho \mathcal{V}) g,
$$

where $D_{0}$ is the steady drag corresponding to the velocity $U_{0}$. The above relation can also be made nondimensional in the form

$$
2 \mathrm{Ar}^{2}=\operatorname{Re}^{2} C_{d}(\operatorname{Re})
$$

where $\operatorname{Ar}=\sqrt{\left(m^{*}-\mathcal{V} / d^{2}\right) g d^{3}} / v$ is the so-called Archimedes number, $\operatorname{Re}=U_{0} d / v$ is the Reynolds number and $m^{*}=m / \rho_{f} d^{2}$ expresses the body-to-fluid mass ratio.

\subsection{Small-amplitude deviations around the base solution: the quasi-static model}

We now consider small-amplitude deviations around the mean vertical path described above. We assume the angles $\alpha, \theta$ and $\gamma$ to be small, and consider that the velocity of the body is $\mathbf{U}=\left(U_{0}+u\right) \mathbf{x}_{\mathbf{a}}$ with $u \ll U_{0}$.

To close the model, we need to express the drag, lift, and torque in terms of the unknown $u, \gamma, \alpha, \omega$. Our line of reasoning here is to model the forces and the torque with quasi-static expressions obtained by considering elementary deviations from the equilibrium state. For this purpose it is appropriate to remark that when the velocity $\mathbf{U}$ of the body is constant, the forces exerted on it are equivalent to those exerted on a fixed body placed within a uniform stream $\mathbf{U}_{\mathbf{i}}=-\mathbf{U}$.

We consider three elementary situations. The first of these (Fig. 2a) corresponds to the case where the body velocity is increased by a small amount $u$ with respect to the equilibrium value $U_{0}$, while the body remains at zero incidence. In this case, one expects the drag to increase by an extra quantity $D^{\prime} \approx D_{, u} u$ with $D_{, u}=\partial D / \partial U$. The second elementary situation (Fig. 2b) corresponds to that where the body is held at a nonzero incidence in an incoming flow $U_{0}$. In that case, a lift force and a torque, both proportional to $\alpha$, are expected to occur. Finally the third situation (Fig. 2c) corresponds to the case where the body slowly rotates at a steady rate $\omega$ within the flow. Here again a lift and a torque, both proportionnal to $\omega$, 
are expected to occur. We thus introduce the following force/torque linear model

$$
D=D_{0}+D_{, u} u, \quad L=L_{, \alpha} \alpha+L_{, \omega} \omega, \quad M=M_{, \alpha} \alpha+M_{, \omega} \omega .
$$

Inserting these expressions into (1) and (2) yields a set of four linear differential equations for the unknowns $(u, \alpha, \gamma, \omega)$, namely

$$
\begin{aligned}
& m \dot{u}=-D_{, u} u, \\
& m U_{0} \dot{\gamma}=-D_{0} \gamma-L_{, \alpha} \alpha-L_{, \omega} \omega, \\
& I \dot{\omega}=M_{, \alpha} \alpha+M_{, \omega} \omega \\
& \omega=\dot{\alpha}+\dot{\gamma} \quad(=\dot{\theta}) .
\end{aligned}
$$

It may be noticed that these equations share strong similarities with those used in flight mechanics textbooks when considering the dynamical stability of an airplane in the longitudinal plane (e.g. McCormick, 1995). An important difference, however, is that in our case the first equation and the other three are uncoupled, while in flight dynamics all four equations are fully coupled.

\subsection{Discussion}

The model contains six coefficients that need to be fitted (including the mean drag $D_{0}$ ). In Section 3 , we shall show how these coefficients can be computed accurately from the Navier-Stokes equations considering "quasi-static" body motions. Prior to this, a few comments about these coefficients are in order. The term $D_{, u}$ is the so-called "marginal drag" which measures the drag increase due to an increase in the body velocity. This coefficient is obviously positive. In the Stokes limit, when the drag is proportional to the velocity, one has $D_{, u}=D_{0} / U_{0}$; on the other hand, in the high-Reynolds number case, when the drag increases quadratically with the velocity, one has $D_{, u}=2 D_{0} / U_{0}$. In the general case $D_{0} / U_{0}<D_{, u}<2 D_{0} / U_{0}$.

The coefficient $L_{, \alpha}$ expresses the lift force generated by a constant incidence angle and is central in the aerodynamical context. We anticipate that $L_{\alpha}>0$, the tilting of the body making pressure forces redirect in the $\mathbf{y}_{\mathbf{a}}$ direction, at least for thin bodies. The coefficient $M_{\alpha}$ is associated with the torque exerted at the body geometrical center in presence of a nonzero incidence. In the present context the coefficient $M_{\alpha}$ will turn out to be negative, indicating that the torque has a stabilizing effect. This contrasts with the case of slender wings where this torque is known to be destabilizing.

The coefficient $L_{, \omega}$ corresponds to the lift exerted on a body rotating at steady rate, i.e. to the well-known Magnus effect. Inviscid theory predicts $L_{, \omega}<0$, so that a body rotating in the positive direction feels a lift force in the $-\mathbf{y}_{\mathbf{a}}$ direction. The inviscid theory is also of little use here, but the property $L_{, \omega}<0$ is still found to hold. Finally, the coefficient $M_{\text {, } \omega}$ corresponds to the torque exerted on a body rotating at steady rate. Such a torque is due to viscous friction and can be $a$ priori expected to be negative in most situations.

\subsection{Eigenmode solutions in the large-mass limit}

We now seek the solutions of the model in eigenmode form and first consider the equation for $u$ which obviously admits an exponentially decaying solution $u=\hat{u} e^{\lambda_{B V V} t}$, where the eigenvalue is

$$
\lambda_{\text {BTV }}=-\frac{1}{m} D_{, u} .
$$

In nondimensional form, this eigenvalue may be expressed as

$$
\lambda_{B T V}^{*}=\frac{d}{U_{0}} \lambda=-\frac{C_{d *}}{2 m^{*}} \quad \text { with } C_{d *}=2 C_{d}+R e \frac{\partial C_{d}}{\partial \operatorname{Re}} .
$$

The physical significance of this mode is clear: if the body velocity departs from the equilibrium value $U_{0}$, the variation of the drag tends to bring it back to $U_{0}$. This is why we call this mode the "back-to-terminal-velocity" mode (BTV).

We now consider the other three coupled degrees of freedom. Again we look for eigenmode solutions in the form $[\alpha, \gamma, \omega]=[\hat{\alpha}, \hat{\gamma}, \hat{\omega}] e^{\lambda t}$. Introducing these expressions in the model yields a $3 \times 3$ system which has nontrivial solutions only if its determinant is zero. This leads to the characteristic equation

$$
m I U_{0} \lambda^{3}+\left[I\left(D_{0}-L_{, \alpha}\right)-m U_{0} M_{, \omega}\right] \lambda^{2}+\left[\left(L_{, \alpha}-D_{0}\right) M_{, \omega}-\left(L_{, \omega}+m U_{0}\right) M_{, \alpha}\right] \lambda-D_{0} M_{, \alpha}=0 .
$$

The next step consists in making (13) dimensionless, considering the limit $m^{*} \gg 1$, and expanding the three roots in powers of $m^{*}$. It is found that one of these roots is real and negative and reads at leading order

$$
\lambda_{B V}=-\frac{D_{0}}{m U_{0}}+O\left(m^{-2}\right) \text { or in dimensionless form } \lambda_{B V}^{*}=-\frac{C_{d}}{2} m^{*-1}+O\left(m^{*-2}\right)
$$

It is noteworthy that this eigenvalue can be retrieved directly from (8) by neglecting the terms proportional to $L, \alpha$ and $L, \omega$. Indeed, performing a more rigorous asymptotic analysis of this solution confirms that, compared to the $\hat{\gamma}$ component, the $\hat{\alpha}$ 
and $\hat{\omega}$ components are negligible at leading order. This solution thus corresponds to a motion in which the trajectory comes back to the vertical while the body axis remains aligned with its path. We call this mode "Back-to-Vertical" (BV).

If $M_{, \alpha} \neq 0$, the two remaining eigenvalues are complex conjugates, indicating an oscillating mode with low frequency (thus noted LF), and have to be expanded in powers of $m^{-1 / 2}$. The first two terms provide the leading contribution to the imaginary and real parts, respectively, and read

$$
\lambda_{L F}= \pm \sqrt{\frac{M_{, \alpha}}{I}}+\left(\frac{L_{, \alpha}}{2 m U_{0}}+\frac{M_{, \omega}}{2 I}\right)+O\left(m^{-3 / 2}\right)
$$

or in dimensionless form

$$
\lambda_{L F}^{*}= \pm i \sqrt{\left|C_{m, \alpha}\right| / 4 C_{I}} m^{*-1 / 2}+\left(C_{l, \alpha} / 4+C_{m, \omega} / 8 C_{I}\right) m^{*-1}+O\left(m^{*-3 / 2}\right) .
$$

Again the leading-order expression of this eigenvalue can be retrieved directly from (9) by neglecting rotation effects and assuming that $\alpha \equiv \theta$ (hence $\gamma \equiv 0$ ). Therefore this mode corresponds to an oscillation of the body inclination along a nearly vertical path.

A particular case that requires a specific treatment is that of the circular cylinder. In this case, the incidence angle $\alpha$ is not a relevant parameter, as the steady problem is invariant by any rotation of angle $\alpha$, implying that $L_{, \alpha}$ and $M_{, \alpha}$ are zero. Considering the characteristic polynomial, it turns out that one of the eigenvalues is zero and is associated with the invariance with respect to $\alpha$. The other two eigenvalues are real and negative. One of them is still given at leading order by (14). The last one is

$$
\lambda_{S P}=\frac{M_{, \omega}}{I}+\mathcal{O}\left(m^{-2}\right) \text { or in dimensionless form } \lambda_{S P}^{*}=\frac{C_{m, \omega}}{4 C_{I}} m^{*-1}+O\left(m^{*-2}\right)
$$

This eigenvalue corresponds to a "spinning mode" with the cylinder spinning about its axis at a rotation rate $\omega$ which is slowly decaying owing to viscous friction.

\section{Computation of the coefficients}

We now turn to the computation of the coefficients $D_{0}, D_{, u}, L_{, \alpha}, L_{, \omega}, M_{, \alpha}$ and $M_{, \omega}$ as function of the fluid flow around the body.

Let $[\mathbf{V}, P]$ be the velocity and pressure fields in this flow. When expressed in the body reference frame which both translates and rotates with respect to the Galilean reference frame, the Navier-Stokes equations take the form

$$
\boldsymbol{\nabla} \cdot \mathbf{V}=0
$$

$$
\frac{\partial \mathbf{V}}{\partial t}+\mathbf{a}_{f}=-(\mathbf{V} \cdot \nabla) \mathbf{V}-\frac{1}{\rho_{f}} \boldsymbol{\nabla} P+v \nabla^{2} \mathbf{V}
$$

with

$$
\mathbf{a}_{f}=\left(\frac{d \mathbf{U}}{d t}+\mathbf{\Omega} \wedge \mathbf{U}+\frac{d \mathbf{\Omega}}{d t} \wedge \mathbf{r}+\mathbf{\Omega} \wedge(\mathbf{\Omega} \wedge \mathbf{r})+2 \mathbf{\Omega} \wedge \mathbf{V}\right) .
$$

where the first two terms in $\mathbf{a}_{f}$ correspond to the acceleration of the body center of inertia expressed in the rotating axes while the other three terms correspond to the so-called Euler entrainment acceleration, centrifugal acceleration and Coriolis acceleration, respectively.

The relative velocity is zero on the body surface while the absolute velocity vanishes at infinity, so that

$$
\begin{aligned}
& \mathbf{V}+(\mathbf{U}+\boldsymbol{\Omega} \wedge \mathbf{r})=0 \quad \text { as }|\mathbf{r}| \rightarrow \infty \\
& \mathbf{V}=0 \quad \text { on } \mathcal{S} .
\end{aligned}
$$

The force and torque exerted on the body are projected onto the body axes in the form

$$
F_{x}=\left(\int_{\mathcal{S}} \mathbf{\Sigma} \cdot \mathbf{n} d S\right) \cdot \mathbf{x}_{\mathbf{b}}, \quad F_{y}=\left(\int_{\mathcal{S}} \boldsymbol{\Sigma} \cdot \mathbf{n} d S\right) \cdot \mathbf{y}_{\mathbf{b}}, \quad M=\left(\int_{\mathcal{S}} \mathbf{r} \wedge(\boldsymbol{\Sigma} \cdot \mathbf{n}) d S\right) \cdot \mathbf{z}
$$

where $\boldsymbol{\Sigma}=-P \mathbf{I d}+\rho_{f} v\left(\boldsymbol{\nabla} \mathbf{V}+{ }^{t} \boldsymbol{\nabla} \mathbf{V}\right)$ denotes the stress tensor (Id being the identity tensor).

As long as the body moves at constant speed $\mathbf{U}$ and does not rotate, the problem (18) and (19) is equivalent to that of the flow past a fixed body placed in the uniform stream of velocity $\mathbf{U}_{i}=-\mathbf{U}$. We first make use of this property by considering the situation where the body falls vertically at zero incidence with the mean velocity $U_{0}$. The corresponding velocity and pressure fields, say $\mathbf{V}_{\mathbf{0}}(x, y)$ and $P_{0}(x, y)$, are the solution of the above Navier-Stokes problem with the left-hand side of (18) set to zero and the boundary condition at infinity $\mathbf{V}_{\mathbf{0}} \rightarrow \mathbf{U}_{i}=U_{0} \mathbf{x}_{\mathbf{b}}$. The mean drag $D_{0}$ is deduced from this solution through the first of (21). 
To compute the other coefficients of the model, we consider three elementary situations, sketched in Fig. 2, which are all small deviations from this reference state. In all three cases the flow field $[\mathbf{V}, P]$ is expanded in the form

$$
[\mathbf{V}, P]=\left[\mathbf{V}_{\mathbf{0}}(x, y), P_{0}(x, y)\right]+[\mathbf{v}(x, y, t), p(x, y, t)],
$$

where perturbations are assumed of small amplitude compared to the base flow. The flow $[\mathbf{v}(x, y, t), p(x, y, t)]$ is governed by the linearized Navier-Stokes equations

$$
\boldsymbol{\nabla} \cdot \mathbf{v}=0
$$

$$
\partial_{t}(\mathbf{v}+\mathbf{u}+\omega \mathbf{Z} \wedge \mathbf{r})+\omega \mathbf{Z} \wedge\left(2 \mathbf{V}_{\mathbf{0}}+\mathbf{U}_{\mathbf{0}}\right)=-(\mathbf{v} \cdot \nabla) \mathbf{V}_{\mathbf{0}}-\left(\mathbf{V}_{\mathbf{0}} \cdot \nabla\right) \mathbf{v}-\frac{1}{\rho_{f}} \nabla p+v \nabla^{2} \mathbf{v}
$$

The first elementary situation (Fig. 2a) corresponds to the case where the velocity of the body slightly differs from the equilibrium velocity: $\mathbf{U}_{i}=\left(U_{0}+u\right) \mathbf{x}_{b}$ with $u \ll U_{0}$. In this case, the flow disturbance can be expected to be steady and proportional to the velocity perturbation $u$ : say, $[\mathbf{v}, p]=u\left[\mathbf{v}^{(u)}, p^{(u)}\right]$. Clearly, the flow field $\left[\mathbf{v}^{(u)}, p^{(u)}\right]$ is the solution of the linearized system (22) and (23) where the time derivative and rotation terms are dropped, and the boundary condition at infinity is selected as $\mathbf{v}^{(u)}=\mathbf{x}_{\mathbf{b}}$. Once this problem is solved, the marginal drag $D_{, u}$ is computed by injecting the stress tensor corresponding to $\left[\mathbf{v}^{(u)}, p^{(u)}\right]$ into the first of (21).

The second elementary situation (Fig. 2b) corresponds to the case where the body has a small, constant incidence $\alpha$ with respect to its path. In the body axes, this is equivalent to considering an incident flow $\mathbf{U}_{i}=-U_{0} \mathbf{x}_{\mathbf{a}} \equiv U_{0} \mathbf{x}_{\mathbf{b}}-U_{0} \alpha \mathbf{y}_{\mathbf{b}}$. The

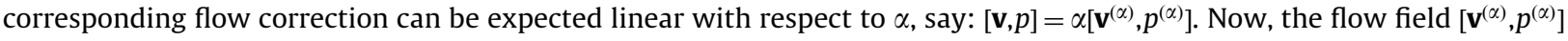
is the solution of the linearized Navier Stokes Eqs. (22) and (23) where the time derivative and rotation terms are still dropped and the boundary condition at infinity is selected as $\mathbf{v}^{(\alpha)}=-\mathbf{y}_{\mathbf{b}}$. Once this problem is solved, the corresponding torque computed through the last of (21) directly provides the coefficient $M_{, \alpha}$ of the model. The corresponding lateral force, say $F_{y}^{(\alpha)}$, is obtained through the second of (21). The lift force is deduced from the axial and lateral forces through $L=F_{y} \cos \alpha-F_{x} \sin \alpha$; at the order $\alpha$ of our expansion this yields $L_{, \alpha}=F_{y}^{(\alpha)}-D_{0}$.

The final elementary situation to consider (Fig. 2c) corresponds to the case where the body is slowly rotating at a steady rate $\omega \ll 1$, within a uniform flow of constant direction. Thus, the incidence angle is increasing in time like $\alpha=\omega t$. This situation is a bit more subtle, as the corresponding flow is not a steady solution of the linearized equations anymore. However, the solution may be expanded in the form $[\mathbf{v}, p]=\omega t\left[\mathbf{v}^{(\alpha)}, p^{(\alpha)}\right]+\omega\left[\mathbf{v}^{(\omega)}, p^{(\omega)}\right]$, where $\left[\mathbf{v}^{(\alpha)}, p^{(\alpha)}\right]$ is the already computed flow structure corresponding to constant incidence, and $\left[\mathbf{v}^{(\omega)}, p^{(\omega)}\right]$ is an additional contribution accounting for rotation. Obviously this approximation is only valid at short time. Injecting this ansatz into (23) yields a linear system which allows computation of $\left[\mathbf{v}^{(\omega)}, p^{(\omega)}\right]$. The latter is then used to compute the parameters $L_{, \omega}$ and $M_{\text {, } \omega}$ of the model through the last two of (21). Note that in the case of a circular cylinder, an alternative method is to consider the steady flow about the body in the inertial frame. Both methods have been used and obviously provide similar results.

The numerical method used to compute the base flow and its corrections in the three elementary situations described above is essentially similar to that employed by Assemat et al. (submitted). Briefly, the spatial discretization makes use of the finite element method as implemented in the FreeFem++ software. An unstructured body-fitted grid covering a large fluid domain is employed. The boundary conditions at infinity are imposed at the inlet and lateral boundary of the domain, while a

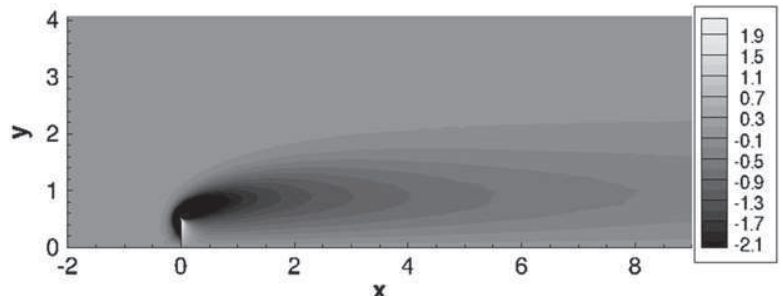

(a)

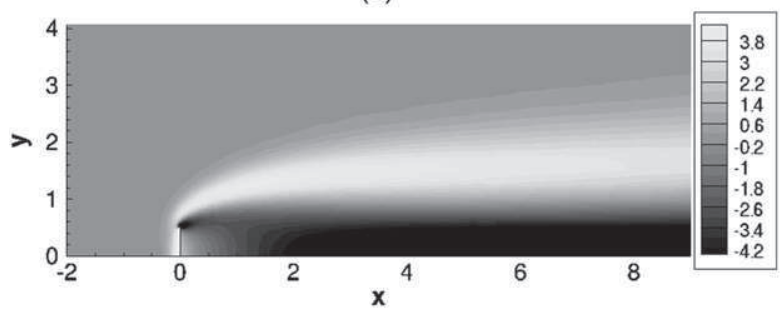

(c)

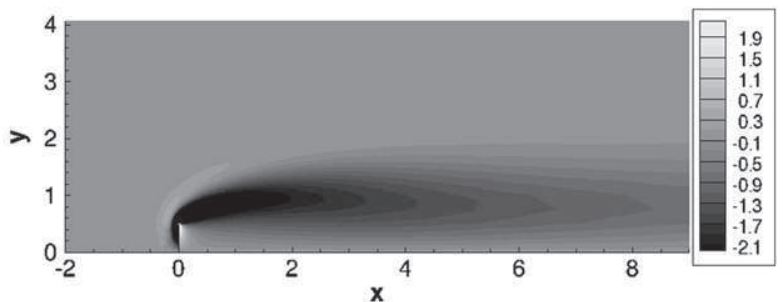

(b)

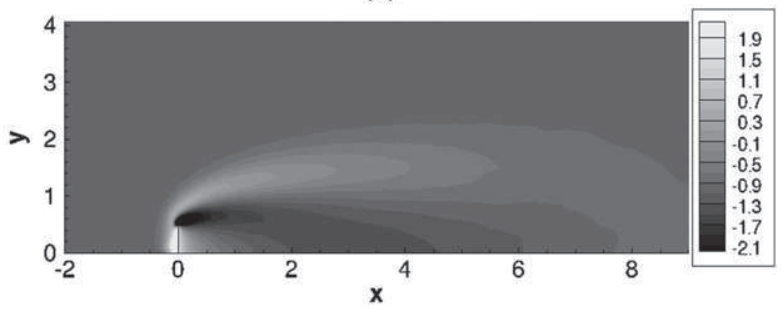

(d)

Fig. 3. Structure of the flow, illustrated by iso-contours of the vorticity, around a flat plate at $R e=30$ : base flow (a); correction due to a variation of velocity $\mathbf{v}^{(\mathbf{u})}(\mathrm{b})$; correction due to a nonzero incidence $\mathbf{v}^{(\boldsymbol{\alpha})}$; correction due to a nonzero rotation $\mathbf{v}^{(\omega)}(\mathrm{d})$. 
weaker condition requiring only the vanishing of the normal derivatives of the velocity components is imposed on the downstream boundary to allow the wake to develop freely. Thanks to the geometrical symmetry of the bodies considered here, it is sufficient to solve the flow field within half-domain, provided suitable symmetry conditions are imposed on the symmetry axis. The base flow, $\left[\mathbf{V}_{\mathbf{0}}, P_{0}\right]$ is computed using a Newton iteration method to reach the solution of the steady nonlinear NavierStokes equations, while each of the three elementary corrections is directly computed as a solution of the corresponding linear problem. All inversions of linear systems are carried out using the library UMFPACK implemented in the software FreeFem++.

As an illustration, Fig. 3 displays the vorticity field in the base flow and its corrections for the three elementary problems considered above in the case of a thin plate with $R e=30$. Because of the obvious symmetry about the vertical direction, only half of the domain is represented. The base flow (3a) exhibits a strong shear layer (with negative vorticity in the $y>0$ halfplane), originating from the edge of the plate. The $u$-correction ( $3 b$ ) has the same global structure, indicating that increasing the body velocity mainly results in an increase of the base vorticity without any qualitative change in its spatial distribution. The correction due to incidence (3c) displays a banded structure with negative (dark) values along the axis and positive (clear) values on each side of the wake. When adding this perturbation to the base-flow, the effect is to shift the shear layer of negative vorticity originating from the edge at $x=0, y=0.5$ towards the $y<0$ direction. Similarly, the shear layer of positive vorticity originating from the edge at $x=0, y=-0.5$ (not shown in Fig. 3a) is also shifted towards the $y>0$ direction. The overall result can be interpreted as a wake developing in the direction of the incoming flow, not aligned with the symmetry plane of the body. The correction due to rotation (3d) mainly affects the vorticity distribution close to the body.

\section{Results for various body shapes}

We now present and comment on the values of the coefficients involved in the model and the computed eigenvalues. We consider three basic geometries, namely a thin plate, a rod with square section and a cylinder with circular section.
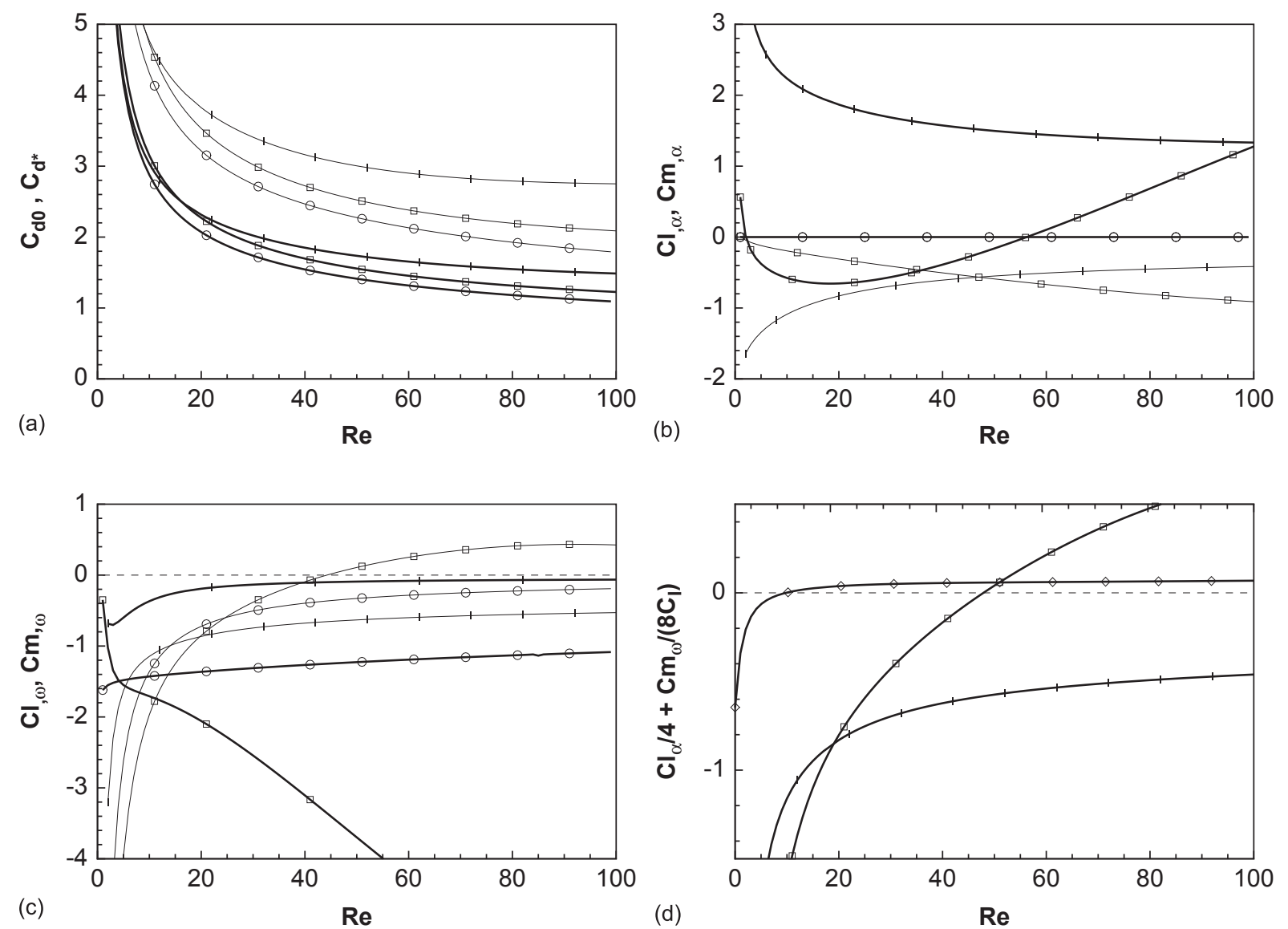

Fig. 4. Coefficients of the model as function of the Reynolds number for a thin plate with uniform loading (I), a square cylinder ( $\square$ ), and a circular cylinder $\left(\bigcirc\right.$ ), respectively. (a) Drag coefficient $C_{d 0}$ (thick lines) and marginal drag coefficient $C_{d^{*}}$ (thin lines); (b) Lift coefficient $C_{l, \alpha}$ (thick lines) and torque coefficient $C_{m, \alpha}$ (thin lines) due to incidence; (c) Lift coefficient $C_{l, \omega}$ (thick lines) and torque coefficient $C_{m, \omega}$ (thin lines) due to rotation; (d) Coefficient giving the real part of the eigenvalue $\lambda_{L F}^{*}$ of the low-frequency quasi-static mode (here the curve identified with the symbol $\diamond$ corresponds to a thin plate loaded at both extremities). 
Fig. 4(a) shows the drag coefficients $C_{d, 0}$ and marginal drag coefficients $C_{d}^{*}=2 C_{d, 0}+\operatorname{Re} \partial C_{d} / \partial R e$. As expected in this intermediate range of $R e$ where the drag evolves like $U^{m}$ with $1<m<2, C_{d}{ }^{*}$ lies between $C_{d 0}$ and $2 C_{d 0}$.

Fig. 4(b) displays the results for the lift and torque coefficients due to incidence, i.e. $C_{l, \alpha}$ and $C_{m, \alpha}$ as function of the Reynolds number for $R e<100$. While these coefficients are obviously zero for a circular cylinder, the torque coefficient $C_{m, \alpha}$ is negative for the other two geometries, indicating that the torque acts to tilt the body back to normal incidence. In the vocabulary of flight dynamics, this means that all bodies are statically stable with respect to a variation of the angle of incidence. Note that potential flow theory predicts $C_{m, \alpha}=4\left(m_{x x}-m_{y y}\right) / \rho_{f} d^{2}$ where $m_{x x}$ and $m_{y y}$ are the added-masses of the body along its principal directions. This quantity is zero in the case of a square cylinder and reaches a value of $4 \pi$ in the limit of an infinitely thin plate. Present low-to-moderate Re results obtained by considering a static incidence significantly differ from this prediction. In the case of a thin plate, the lift coefficient $C_{l, \alpha}$ is positive whatever the Reynolds number. For this particular shape, the positive lift is mainly due to the pressure forces normal to the plate which provide a force component perpendicular to the body axis. On the other hand, it can be noticed that in the case of a square rod, the liftdue-to-incidence is negative for $R e \leq 68$ and positive for higher Reynolds numbers. This point was already noticed by Sohankar et al. (1998) who argued that the pressure and viscous shear contributions to the lift have opposite signs for this geometry.

Fig. 4 (c) displays the values of the lift and torque coefficients due to rotation, i.e. $C_{l, \omega}$ and $C_{m, \omega}$. The lift coefficient $C_{l, \omega}$ is negative whatever the body shape. This is consistent with the classical explanation of the Magnus effect according to which a positive body rotation accelerates (resp. decelerates) the flow and hence decreases (resp. increases) the pressure on its lower (resp. upper) part, thus resulting in a net downward force. Again, potential flow theory predicts $C_{m, \omega}=4 \pi$ for a circular cylinder, which is far from the values found within the range of Reynolds numbers considered here. The torque coefficient $C_{m, \omega}$ is always negative in the case of a thin plate, indicating that viscous friction opposes the rotation. On the other hand, this contribution is found to be positive in the case of a square cylinder for $R e \geq 44$.

Finally, the most important prediction of the model is synthesized in Fig. 4(d) which displays the normalized real part of the eigenvalue $\lambda_{L F}$ of the low-frequency oscillating mode for the case of a thin plate (with two different distributions of mass) and a square rod. In the case of a plate with uniform mass loading, the model predicts that the system is stable whatever the Reynolds number in the range considered. Hence it turns out that the present model is unable to explain how fluttering, which is commonly observed in experiments involving heavy plates, could emerge from the rectilinear motion through a Hopf bifurcation. Implications of this result will be discussed in Section 6. As indicated in (16), the real part of the eigenvalue contains two terms: the lift-due-to-incidence, which is destabilizing, and the torque-due-to-rotation, which is stabilizing. The overall negative sign indicates that the second term dominates for a thin plate with a uniform loading. In contrast, a different mass loading may modify this balance. As a matter of fact, in the extreme case where the mass is entirely located at the tips of the plate (which corresponds to $c_{I}=1 / 4$ ), the amplification rate crosses zero at $R e \approx 12$ and remains weakly positive beyond this value (this situation is indicated with diamonds in Fig. 4(d)).

The case of a square rod with uniform mass loading yields very different results. Then the amplification rate crosses zero for $R e \approx 48$ and reaches significant positive values for higher $R e$. It is noteworthy that in this case, both terms involved in the amplification rate change sign as the Reynolds number is increased. Hence, at the threshold value $R e \approx 48$, the liftdue-to-incidence is stabilizing while the torque-due-to-rotation is destabilizing.

\section{Comparison with results of a global stability study}

In a companion paper (Assemat et al., submitted), we carry out a global stability analysis of the coupled fluid/body system. Here we compare the results obtained through this more general approach with the predictions of the present model (see Table 1). For the purpose of validation of the model, we consider the above three geometries with a

Table 1

Comparison of eigenvalues obtained with the present model with those provided by the global stability code of Assemat et al. (submitted) for various bodies in the case $m^{*}=1000, R e=50$.

\begin{tabular}{llll}
\hline Body shape & Mode & Eigenvalue & Large- $m^{*}$ prediction \\
\hline Circular cylinder & BTV & -0.001121 & -0.001125 \\
& BV & -0.0006912 & -0.0006986 \\
& Spin & -0.0006615 & -0.0006575 \\
& VK & $0.01422 \pm 0.7437 \mathrm{i}$ & - \\
Plate (uniform loading) & BTV & -0.001368 & -0.001382 \\
& BV & -0.0008135 & -0.0008129 \\
LF & VK & $-0.000616 \pm 0.03984 \mathrm{i}$ & $-0.000613 \pm 0.03983 \mathrm{i}$ \\
Square rod & BTV & $0.0817 \pm 0.5846 \mathrm{i}$ & -0.001281 \\
& BV & -0.001205 & -0.0007897 \\
& LF & -0.0007906 & $0.000047 \pm 0.03009 \mathrm{i}$ \\
\hline
\end{tabular}


dimensionless mass $m^{*}=1000$ at the single Reynolds number $R e=50$. Comparisons over a wider range of $R e$ and $m^{*}$ are presented in Assemat et al. (submitted). Table 1 shows that all the eigenvalues predicted by the global stability approach are captured by the present model with a very good accuracy.

In addition, the global approach reveals the existence of a pair of unstable complex eigenvalues, with imaginary parts much larger than those associated with the LF quasi-static mode. This pair of eigenvalues actually corresponds to the wellknown von Kármán (VK) vortex shedding mode which is unstable for $R e=50$ for all three geometries considered here. In the case of very heavy bodies, we expect that this von Kármán mode has almost no effect on the path of the body, and so manifests itself in the same way as in the case of fixed bodies embedded in a uniform flow (these expectations are indeed confirmed in Assemat et al., submitted).

\section{Summary and discussion}

The goal of this study was to investigate the motion of two-dimensional bodies falling freely under the effect of gravity within a viscous fluid, in order to get new insight on the onset of the commonly observed fluttering motion. By restricting ourselves to cases where deviations with respect to the vertical path are small, we derived a linear system of four coupled differential equations that govern the velocity disturbance (with amplitude $u$ and direction $\gamma$ ), the body inclination $\theta$ and rotation rate $\omega$, respectively. The forces and the torque acting on the body were modelled through a quasi-static approach by considering elementary situations in which the body falls with a fixed orientation, or rotates at a very slow rate. This modelling approach is justified as far as the trajectory evolves on a long time scale with respect to that associated with the flow, a condition fulfilled in the case of very heavy bodies. In addition to two eigenmodes which are always damped, this approach reveals the existence of a low-frequency oscillating eigenmode which could be related to the fluttering motions observed at larger values of the Reynolds number. However, for slender bodies such as a thin plate with a uniform mass loading, this mode is found to be linearly stable throughout the range of Reynolds numbers we considered.

These findings indicate that, contrary to our initial expectations, the onset of fluttering cannot be explained as the result of a supercritical Hopf bifurcation from the steady vertical path, at least in the high-mass limit in which our model is justified. A possible explanation to this puzzle could be that, as soon as the von Kármán vortex shedding sets in, the base flow around which our expansion is not observed any more. Thus, fluttering could actually occur through a secondary Hopf bifurcation from a quasi-vertical path in which vortex shedding is already present and has reached its saturation amplitude. This hypothesis could be tested by repeating the present analysis with a base flow taken as the mean flow, obtained by a time average over one period of vortex shedding, rather than with the unstable base flow as considered here (Sipp and Lebedev, 2007). Note that for $R e \geq 100$ the base flows as considered here become pretty not realistic (in particular they display a recirculation region much larger than the body diameter, in contradiction with all observations in this range). This is why we restricted the present analysis to lower Reynolds numbers.

Other hypotheses may be considered. For instance, the bifurcation to the fluttering mode could be strongly subcritical, thus making any attempt to explain it by considering linear expansions around a vertical path hopeless. Or, the fluttering motions may be of a different nature compared to the low-frequency oscillation modes evidenced here.

Finally, a positive result of the present study is the prediction of the existence of an unstable low-frequency oscillating mode for thicker bodies or thin bodies with nonuniform mass distributions. For instance, for a square cylinder, the model predicts that this low-frequency mode becomes unstable for $R e \approx 48$. Interestingly, this threshold value is very close to that corresponding to the onset of the von Kármán vortex shedding mode. Rich dynamics resulting from the coupling of both modes can thus be expected.

Direct numerical simulations of the free motion of two-dimensional bodies are in progress in our team, and should soon provide answers to the questions raised above. Extensions of both the present model and the global stability approach to the case of three-dimensional, axisymmetric bodies such as disks, cylinders of finite span or bubbles is also in progress.

\section{Acknowledgements}

This study was supported by the French National Agency for Research (ANR) in the framework of the OBLIC project. The authors thank Paolo Luchini for stimulating discussions.

\section{References}

Andersen, A., Pesavento, U., Wang, Z.J., 2005a. Analysis of transitions between fluttering, tumbling and steady descent of falling cards. J. Fluid. Mech. 541, 91-104.

Andersen, A., Pesavento, U., Wang, Z.J., 2005b. Unsteady aerodynamics of fluttering and tumbling plates. J. Fluid Mech. 541, 65-90.

Belmonte, A., Eisenberg, H., Moses, E., 1998. From flutter to tumble: inertial drag and froude similarity in falling paper. Phys. Rev. Lett. 81, $345-348$.

Ern, P., Risso, F., Fernandes, P.C., Magnaudet, J., 2009. A dynamical model for the buoyancy-driven zigzag motion of oblate bodies. Phys. Rev. Lett. 102, 134505.

Fernandes, P., Risso, F., Ern, P., Magnaudet, J., 2007. Oscillatory motion and wake instability of freely-rising axisymmetric bodies. J. Fluid Mech. 573, 479-502.

Field, S., Klaus, M., Moore, M., Nori, F., 1997. Chaotic dynamics of falling disks. Nature 388, 252-254. 
Horowitz, M., Williamson, C., 2010. The effect of Reynolds number on the dynamics and wakes of freely rising and falling spheres. J. Fluid. Mech. 651, $251-294$

Jin, C., Xu, K., 2008. Numerical study of the unsteady aerodynamics of the freely falling plates. Commun. Comput. Phys. 3, 834-851.

Jones, M.A., Shelley, M.J., 2005. Falling cards. J. Fluid Mech. 540, 393-525.

Kolomenskiy, D., Schneider, K., 2010. Numerical simulations of falling leaves using a pseudo-spectral method with volume penalization. Theor. Comput. Fluid Dyn. 24, 169-173.

Lamb, S.H., 1945. Hydrodynamics, sixth ed. Dover.

Lugt, H., 1983. Autorotation. Annu. Rev. Fluid. Mech. 15, 123-147.

Mahadevan, L., 1996. Tumbling of a falling card. C. R. Acd. Sci. Paris 323, 729-736.

Mahadevan, L., Ryu, W.S., Samuel, A.D.T., 1999. Tumbling cards. Phys. Fluids 11, 1-3.

Maxwell, J.C., 1854. On a particular case of the descent of a heavy body in a resisting medium. Camb. Dublin Math. J. 9, 145-148.

McCormick, B.W., 1995. Aerodynamics, Aeronautics, and Flight Mechanics. Wiley.

Pesavento, U., Wang, Z.J., 2004. Falling paper: Navier-Stokes solutions, model of fluid forces, and center of mass elevation. Phys. Rev. Lett. 93, 144501.

Sipp, D., Lebedev, A., 2007. Global stability of base and mean flows: a general approach and its applications to cylinder and open cavity flows. J. Fluid Mech. 593, 333-358.

Sohankar, A., Norberg, C., Davidson, L., 1998. Low-Reynolds flow around a square cylinder at incidence: study of blockage, onset of vortex shedding and open boundary condition. Int. J. Numer. Meth. Fluids 26, 39-56.

Tanabe, Y., Kaneko, K., 1994. Behavior of a falling paper. Phys. Rev. Lett. 73, 1372-1375.

Willmarth, W.W., Hawk, N.E., Harvey, R.L., 1964. Steady and unsteady motions and wakes of freely falling disks. Phys. Fluids 7, 197-208. 